hep-th/9912226

KCL-MTH-99-49

\title{
Brane Rotating Symmetries And the Fivebrane Equations of Motion
}

\author{
Oliver Bärwald円, and Peter C. West \\ oliver, pwest@mth.kcl.ac.uk \\ Department of Mathematics, King's College London \\ Strand, London WC2R 2LS, Great Britain
}

July 27, 2021

\begin{abstract}
We show that the fully covariant equations of motion for the Mtheory fivebrane can be interpreted as charge conservation equations. The associated charges induce 'shift'-symmetries of the scalar, spinor and gauge-fields of the fivebrane, so allowing an interpretation of all these fields as Goldstone fields. We also find that the fivebrane possesses a new symmetry that is part of the $G L(32)$ automorphism group of the eleven dimensional supersymmetry algebra.
\end{abstract}

In this paper we give a new interpretation to the fivebrane equations of motion. We show that these can be written as conservation equations for three conserved currents. The symmetries generated by the associated charges correspond to constant 'shifts' of the scalar, spinor and gauge-fields respectively.

We then compute the algebra generated by these charges. This turns out to be - as expected the eleven dimensional supersymmetry algebra with two-form and five-form central charges present. Hence in this manner we have reconstructed the eleven dimensional supersymmetry from the fivebrane equations of motion. This superalgebra has previously been derived [8] in the context of the Lagrangian formulation of the fivebrane. By reducing the eleven dimensional supersymmetry algebra, we find the $(2,0)$ worldvolume supersymmetry algebra. The currents that give rise to this worldvolume superalgebra are explicitly constructed.

We also find that the fivebrane contains a new symmetry that is part of the $G L(32)$ automorphism group of the eleven dimensional supersymmetry algebra. This symmetry rotates the fivebrane and twobrane into each other. Finally, we discuss some of the possible consequences for $\mathrm{M}$ theory of this new symmetry.

\footnotetext{
${ }^{1}$ Supported by the EC under TMR contract ERBFMBICT972717,
} 


\section{Conserved Currents and the Equations of Motion}

The fivebrane-equations of motion for a flat background and ignoring higher order terms in the fermion fields are given by [6]

$$
\begin{aligned}
G^{m n} \nabla_{m} \nabla_{n} X^{\underline{a}} & =0 \\
\nabla^{m} \Theta(1-\Gamma) \Gamma^{n} m_{m n} & =0 \\
G^{m n} \nabla_{m} H_{n p q} & =0
\end{aligned}
$$

We will now define the symbols occurring in the equations of motion: Our index-convention is to use $m, n, p, \ldots=0,1, \ldots, 5$ and $a, b, c, \ldots=0,1, \ldots, 5$ for world-volume world and tangent indices respectively. For the flat target-space indices we use $\underline{a}, \underline{b}, \underline{c}, \ldots=0,1, \ldots, 10$. In static gauge the targetindices can be decomposed into world-volume and transverse indices as $\underline{a}=\left(a, a^{\prime}\right)$. For fermionic indices it is customary to use same scheme with Greek letters, however we are going to suppress these indices in most of the following for simplicity. We will use the 'mostly plus' convention for the Minkowski-metric $\eta_{a b}:=\operatorname{diag}(-1,1, \ldots, 1)$ and normalize the $\epsilon$-tensor as $\epsilon^{012345}=1$.

The metric on the brane is the pullback of the flat target space metric

$$
g_{m n}:=\partial_{m} X^{\underline{a}} \partial_{n} X^{\underline{b}} \eta_{\underline{a b}},
$$

and the covariant derivative is defined with the Levi-Civita connection with respect to this metric. We define the associated vielbein in the standard way as

$$
e_{m}{ }^{a} e_{n}{ }^{b} \eta_{a b}:=g_{m n}
$$

It will be necessary to switch frequently between frames. To make life simpler we will therefore normally suppress the vielbein-factors.

The tensor $G^{m n}$ is related to the induced metric via

$$
G^{m n}:=\left(m^{2}\right)^{m n},
$$

where the matrix $m$ is given in tangent frame as

$$
m_{a b}:=\eta_{a b}-2 h_{a}{ }^{c d} h_{b c d} \equiv \eta_{a b}-2 k_{a b} .
$$

The 3 -form field $h_{a b c}$ is self-dual

$$
h_{a b c}=\frac{1}{3 !} \epsilon_{a b c d e f} h^{d e f},
$$

but it is not the curl of a two-form gauge field. It is related to the field $H_{m n p}=3 \partial_{[m} B_{n p]}$ which appears in the equations of motion, but $H_{m n p}$ is not self-dual in the linear sense. The relationship between the two fields is given by

$$
H_{a b c}=\left(m^{-1}\right)_{c}{ }^{d} h_{a b d} .
$$

Finally the matrix $\Gamma$ appearing in the equation of motion for the fermions is given by

$$
\Gamma=-\frac{1}{6 !} \frac{1}{\sqrt{-g}} \epsilon^{m_{1} \ldots m_{6}} \Gamma_{m_{1} \ldots m_{6}}+\frac{1}{3} h^{m n p} \Gamma_{m n p} .
$$


It satisfies $\operatorname{Tr} \Gamma=0$ and $\Gamma^{2}=1$ and hence gives rise to the projector $\frac{1}{2}(1-\Gamma)$, projecting onto half of the original spinor space.

The covariantly conserved energy-momentum tensor for this system is given by $T^{m n}=Q^{-1} G^{m n}$ [2] where $Q=1-\frac{2}{3} k^{a b} k_{a b}$. Using this we can rewrite (11) as

$$
0=T^{m n} \nabla_{m} \partial_{n} X^{\underline{a}}=\nabla_{m}\left(T^{m n} \partial_{n} X^{\underline{a}}\right)=\frac{1}{\sqrt{-g}} \partial_{m}\left(\sqrt{-g} T^{m n} \partial_{n} X^{\underline{a}}\right) .
$$

We can interpret this as the condition that the current

$$
J_{X}^{m \underline{a}}:=\sqrt{-g} T^{m n} \partial_{n} X^{\underline{a}}
$$

is conserved.

Let us now turn to the equation of motion for the fermions. Here we have to do a bit more work. Using $\nabla_{m}\left(Q^{-1} m^{m n}\right)=0$ [7] we can rewrite (2) as

$$
\begin{aligned}
0 & =Q^{-1} m^{m n} \nabla_{m} \Theta(1-\Gamma) \Gamma_{n} \\
& =\nabla_{m}\left(Q^{-1} m^{m n} \Theta(1-\Gamma) \Gamma_{n}\right) \\
& =\frac{1}{\sqrt{-g}} \partial_{m}\left(\sqrt{-g} Q^{-1} m^{m n} \Theta(1-\Gamma) \Gamma_{n}\right)
\end{aligned}
$$

In the second step we used $m^{m n} \nabla_{m}\left((1-\Gamma) \Gamma_{n}\right)=0$. To see this first note that $\nabla_{m} \Gamma_{n}=0$. This follows from the fact that the curved $\Gamma_{m}$ are constructed from the flat 11-dimensional $\Gamma_{\underline{a}}$ as $\Gamma_{m}=\partial_{m} X \underline{a} \Gamma_{\underline{a}}$ and the covariant derivatives $\nabla_{m}$ are associated with the induced metric $g_{m n}=\partial_{m} X \underline{a} \partial_{n} X \underline{b} \eta_{a b}$. It remains to show that $m^{m n} \nabla_{m}\left(\Gamma_{(h)} \Gamma_{n}\right)=0$. We have, moving to tangent frame,

$$
\begin{aligned}
m^{a b} \nabla_{a} \Gamma_{(h)} \Gamma_{b} & =\frac{1}{3} m^{a b} \nabla_{a} h^{c_{1} c_{2} c_{3}} \Gamma_{c_{1} c_{2} c_{3}} \Gamma_{b} \\
& =\frac{1}{3} m^{a b} \nabla_{a} h^{c_{1} c_{2} c_{3}}\left(\Gamma_{c_{1} c_{2} c_{3} b}+3 \eta_{c_{1} b} \Gamma_{c_{2} c_{3}}\right) \\
& =\frac{1}{3} m^{a b} \nabla_{a} h^{c_{1} c_{2} c_{3}}\left(\frac{1}{2} \Gamma_{012345} \epsilon_{c_{1} c_{2} c_{3} b c_{4} c_{5}} \Gamma^{c_{4} c_{5}}+3 \eta_{c_{1} b} \Gamma_{c_{2} c_{3}}\right) \\
& =m^{a b} \nabla_{a} h_{b c_{2} c_{3}} \Gamma^{c_{2} c_{3}}\left(1-\Gamma_{012345}\right) \\
& =0 .
\end{aligned}
$$

We have used the duality relation for the $\Gamma_{a}$ matrices and in the last step also the equation of motion for the self-dual field-strength $m^{a b} \nabla_{a} h_{b c d}=0$ [6]. Hence again we can identify a conserved current

$$
J_{\Theta}^{m}:=\sqrt{-g} Q^{-1} m^{m n} \Theta(1-\Gamma) \Gamma_{n},
$$

where we suppressed the target-space spinor-indices. Note that due to the presence of the projector $(1-\Gamma)$ we only get 16 independent currents.

Analogously we can rewrite the tensor field equation of motion (3) as

$$
0=T^{m n} \nabla_{m} H_{n}{ }^{p q}=\nabla_{m}\left(T^{m n} H_{n}{ }^{p q}\right)=\frac{1}{\sqrt{-g}} \partial_{m}\left(\sqrt{-g} T^{m n} H_{n}{ }^{p q}\right) .
$$

In the last step we have used that $T^{m n} H_{n}{ }^{p q}=Q^{-1} G^{m n} H_{n}{ }^{p q}$ is totally anti-symmetric in $m, p$ and $q$. To see this recall that $G^{m n}=\left(m^{2}\right)^{m n}$ and $H_{m n p}=\left(m^{-1}\right)_{m}{ }^{q} h_{q n p}$. This gives

$$
T_{n}^{m} H^{n p q}=Q^{-1} m_{n}^{m} h^{n p q}=* H^{m p q},
$$


where $* H^{m p q}$ is the dual of $H_{m p q}$ from which the antisymmetry follows. Again we can identify a conserved current

$$
J_{H}^{m n p}:=\sqrt{-g} T^{m q} H_{q}^{n p}=\sqrt{-g} * H^{m n p} .
$$

\section{Identifying the Symmetries}

Having found three new conserved currents it is natural to ask what symmetries the associated charges will generate. Recall that given any current satisfying $\partial_{m} J^{m}=0$ we can define an associated timeindependent charge

$$
Q:=\int d^{5} x J^{0}
$$

which will generate a symmetry transformation on some field $\Phi$ via

$$
\delta \Phi=\{Q, \Phi\}
$$

where $\{$,$\} denotes the Poisson bracket.$

Unfortunately within the covariant approach to the fivebrane used here we do not have a Lagrangian for the fivebrane fields. However, to identify the symmetries it will however be sufficient to keep only the lowest order terms in the currents and then use the free-field Poisson brackets. We denote these lowest order currents by the same symbol the distinction between them and their complete form being apparent from the context. The lowest order currents are given by

$$
\begin{aligned}
J_{X}^{m \underline{a}} & =\partial^{m} X^{\underline{a}}, \\
J_{H}^{m n p} & =h^{m n p}, \\
J_{\Theta}^{m} & =\Theta(1-\Gamma) \Gamma^{m} .
\end{aligned}
$$

The free-field (equal time) Poisson brackets are given by

$$
\begin{aligned}
\left\{X^{\underline{a}}(x), \partial_{0} X^{\underline{b}}\left(x^{\prime}\right)\right\} & =\eta^{\underline{a b}} \delta\left(x-x^{\prime}\right) \\
\left\{\Theta(x), \Theta\left(x^{\prime}\right)^{\dagger}\right\} & =\delta\left(x-x^{\prime}\right), \\
\left\{B_{i j}, H^{0 l k}\right\} & =\delta_{[i}^{l} \delta_{j]}^{k} \delta\left(x-x^{\prime}\right) .
\end{aligned}
$$

Some comments are in order regarding the last equation where we have used $i, j, \ldots$ to denote purely spatial indices. Since one does not have a simple free action for the self-dual gauge field one can not deduce its Poisson bracket in the standard way. However, in what follows it will be sufficient to adopt the above Poisson bracket for the linearized fields.

Using the explicit Poisson brackets and including constant parameters one readily checks

$$
\left\{\xi_{\underline{b}} \bar{Q}_{X}^{\underline{b}}, X^{\underline{a}}\right\}=\xi_{\underline{b}} \int d^{5} x\left\{\partial^{0} X^{\underline{b}}, X^{\underline{a}}\right\}=\xi^{\underline{a}} .
$$

Hence $\bar{Q}_{X}$ generates 'shifts' of the scalar fields as expected. For the spinors we find

$$
\left\{\bar{Q}_{\Theta} \bar{\epsilon}, \Theta\right\}=\epsilon(1-\Gamma),
$$

again constant 'shifts'. Note that the occurrence of the projection operator implies that only half the Fermions are getting shifted; these are precisely the dynamical Fermions appearing in the Dirac equation. 
Finally for the gauge field we have

$$
\left\{a_{p q} \bar{Q}_{H}^{p q}, B_{m n}\right\}=a_{m n} .
$$

Hence we find that all the fields of the fivebrane undergo shift symmetries and therefore the fivebrane itself can be interpreted as a Goldstone object associated with the superalgebra of the above generators. The way the symmetry generators arise from the equations of motion is also typical of the Goldstone phenomenon.

\section{The Eleven dimensional Superalgebra}

We now want to determine the algebra of the conserved charges using the currents with only their lowest order terms. In this approximation the algebra has only one non-vanishing Poisson bracket which is given by

$$
\begin{aligned}
\left\{\bar{Q}_{\Theta} \bar{\epsilon}_{1}, \bar{Q}_{\Theta} \bar{\epsilon}_{2}\right\}= & 2 \epsilon_{1} \Gamma_{\underline{a}} \bar{\epsilon}_{2} \bar{Q}_{X}^{\underline{a}}+2 \epsilon_{1} \Gamma_{\underline{a}_{1} \ldots \underline{a}_{5}} \bar{\epsilon}_{2} Z_{5} \underline{a}_{1} \ldots \underline{a}_{5} \\
& +2 \epsilon_{1} \Gamma_{\underline{a}_{1} \underline{a}_{2}} \bar{\epsilon}_{2} Z \underline{a}_{2} \underline{a}_{2} .
\end{aligned}
$$

Here $Z_{5}^{\underline{a}_{1} \ldots \underline{a}_{2}}=\int d^{5} x \frac{1}{5 !} \epsilon^{i_{1} \ldots i_{5}} \partial_{i_{1}} X^{a_{1}} \ldots \partial_{i_{5}} X^{a_{5}}$ is the five-form central charge associated with the fivebrane and $Z_{2}^{\underline{a}_{1} \underline{a}_{2}}=\int d^{5} x h^{0 i j} \partial_{i} X^{\underline{a}_{1}} \partial_{j} X_{\underline{a}_{2}}$ is the two-form central charge associated with the membrane. This is the standard 11-dimensional superalgebra with five-form and two-form central charges present. In the fivebrane context it was first given in [8] using the Lagrangian formulation of the fivebrane. We can interpret this result to say that the presence of the fivebrane generates the central charges of the eleven dimensional superalgebra.

In deriving (28) we have used various algebraic properties of the matrices $\Gamma_{(h)}$ and $\Gamma_{(0)}$, these can be found in [1], and also the following Majorana-flip-identity

$$
\psi \Gamma_{\underline{a}_{1} \ldots \underline{a}_{m}} \bar{\phi}=(-1)^{\frac{m(m+1)}{2}} \phi \Gamma_{\underline{a}_{1} \ldots \underline{a}_{m}} \bar{\psi},
$$

which can be derived using the standard properties of the flat eleven dimensional $\Gamma_{\underline{a}}$ matrices.

\section{The Worldvolume Superalgebra}

Having reconstructed the eleven dimensional superalgebra from the equations of motion for the fivebrane we will now make contact with the $(2,0)$ supersymmetry algebra living on the world-volume of the five brane. The fivebrane breaks half of the supersymmetries of the background superspace and its the worldvolume supersymmetry can be found from the eleven dimensional supersymmetry algebra by taking half of the supersymmetry parameter to vanish, namely

$$
\epsilon^{\underline{\alpha}}=\left(\epsilon^{\alpha}, 0\right) \text {. }
$$

Under this truncation the term that contains the momentum becomes

$$
\epsilon_{1} \Gamma_{\underline{a}} \bar{\epsilon}_{2} Q_{X}^{\underline{a}}=\epsilon_{1} \Gamma_{\underline{a}} \Gamma_{0} \epsilon_{2}^{\dagger} Q \underline{a}=\epsilon_{1} \Gamma_{m} \Gamma_{0} \epsilon_{2}^{\dagger} Q_{X}^{m}=\epsilon_{1} \Gamma_{m} \bar{\epsilon}_{2} Q_{X}^{m},
$$

where we have used the explicit eleven dimensional $\Gamma_{\underline{a}}$ matrices given in [3] to identify nonzero contributions. The two central charge terms can be reduced in complete analogy and we find, omitting the supersymmetry parameters and using static gauge,

$$
\left\{Q_{\alpha}^{i}, Q_{\beta}^{j}\right\}=\eta^{i j}\left(\gamma_{m}\right)_{\alpha \beta}\left(P^{m}\right)^{\prime}+\left(\gamma_{m}\right)_{\alpha \beta} Z_{1}^{i j m}+\left(\gamma_{m_{1} m_{2} m_{3}}\right)_{\alpha \beta} Z_{3}^{i j m_{1} m_{2} m_{3}},
$$


with

$$
\begin{aligned}
\left(P^{m}\right)^{\prime} & =\int d^{5} x\left(T^{0 m}-\eta^{0 m}\right), \\
Z_{1}^{i j m} & =-2 \int d^{5} x\left(\left(\gamma_{a^{\prime}}\right)^{i j} h^{0 m n} \partial_{n} X^{a^{\prime}}+\frac{1}{4 !} \gamma_{a_{1}^{\prime} \ldots a_{4}^{\prime}}^{i j} \epsilon^{0 m n_{1} \ldots n_{4}} \partial_{n_{1}} X^{a_{1}^{\prime}} \cdots \partial_{n_{4}} X^{a_{4}^{\prime}}\right), \\
Z_{3}^{i j m_{1} m_{2} m_{3}} & =\frac{1}{3 !} \int d^{5} x\left(\gamma_{a_{1}^{\prime} a_{2}^{\prime}}\right)^{i j} \epsilon^{0 m_{1} m_{2} m_{3} n_{1} n_{2}} \partial_{n_{1}} X^{a_{1}^{\prime}} \partial_{n_{2}} X^{a_{2}^{\prime}} .
\end{aligned}
$$

This is indeed the six-dimensional $(2,0)$ supersymmetry algebra [5] with one-form and three-form central charges present. The internal symmetry group is $U S p(4) \simeq \operatorname{Spin}(5)$, and $\eta^{i j}$ denotes the associated antisymmetric invariant tensor. The Weyl spinor indices $\alpha, \beta, \ldots$ run from 1 to 4 as do the internal Spin(5) indices $i, j, \ldots$ The $\gamma$-matrices arise as building blocks of the flat eleven dimensional $\Gamma^{\underline{a}}$ matrices via

$$
\Gamma^{m}=\delta_{i}^{j}\left(\begin{array}{cc}
0 & \left(\gamma^{m}\right)_{\alpha \beta} \\
\left(\tilde{\gamma}^{m}\right)^{\alpha \beta} & 0
\end{array}\right) \quad \text { and } \quad \Gamma^{a^{\prime}}=\left(\gamma^{\alpha^{\prime}}\right)_{i}{ }^{j}\left(\begin{array}{cc}
\delta_{\alpha}{ }^{\beta} & 0 \\
0 & -\delta_{\beta}^{\alpha}
\end{array}\right) .
$$

We use $m, n, p, \ldots=0,1, \ldots, 5$ and $a^{\prime}, b^{\prime}, c^{\prime}, \ldots=6,7, \ldots, 10$ here to denote flat indices. The basic relations are

$$
\left\{\gamma^{m}, \gamma^{n}\right\}:=\gamma^{m} \tilde{\gamma}^{n}+\gamma^{n} \tilde{\gamma}^{m}=2 \eta^{m n}
$$

with $\tilde{\gamma}^{m}=\gamma^{m}$ for $m \neq 0$ and $-\tilde{\gamma}^{0}=\gamma^{0}=1$. The antisymmetric product is defined as

$$
\gamma^{m_{1} m_{2} m_{3} \cdots}:=\gamma^{\left[m_{1}\right.} \tilde{\gamma}^{m_{2}} \gamma^{m_{3}} \cdots,
$$

and one also has the following duality relation

$$
\gamma^{m_{1} m_{2} \ldots m_{n}}=-\frac{1}{(6-n) !}(-1)^{\frac{n(n+1)}{2}} \epsilon^{m_{1} m_{2} \ldots m_{n} m_{n+1} \ldots m_{6}} \gamma_{m_{n+1} \ldots m_{6}} .
$$

Note that in the integral representation of the momentum $\left(P^{m}\right)^{\prime}$ we find $T^{m n}-\eta^{m n}$ instead of simply $T^{m n}$; the additional term appears in the reduction of $Z_{5}$. Recall that $T^{m n}$ reduces to $\eta^{m n}$ for a flat static brane. However, from the point of view of the world-volume theory this is a vacuum configuration and has to have zero energy in a supersymmetric theory. Hence the tensor that appears in the $\left(P^{m}\right)^{\prime}$ above is therefore the natural energy momentum tensor. Note also that the one-form central charge in six dimensions receives contributions from both the five-form and the two-form charge in eleven dimensions.

We now give the currents that generate the above worldvolume algebra. Clearly, the current $J_{H}^{m n n p}$ associated with the gauge field is a current associated with the worldvolume algebra. The other currents, namely those associated with translations and supersymmetry transformations, can be deduced from their eleven dimensional counter parts by choosing superstatic gauge and considering only those components that lie in the worldvolume directions. One then can then verify that the resulting current transform covariantly and are indeed conserved. For the translations we find that in static gauge we have

$$
J_{X}^{m n}=\sqrt{-g} T^{m p} \partial_{p} X^{n}=\sqrt{-g} T^{m n} .
$$

Which is indeed the conserved current that generates six dimensional translations [2]. 
In superstatic gauge the spinor $\Theta^{\underline{\alpha}}$ takes the form

$$
\Theta^{\underline{\alpha}}=\left(\Theta^{\alpha}, \Theta^{\alpha^{\prime}}\right) .
$$

The linearized six-dimensional supercurrent is given as a straightforward reduction of the eleven dimensional one (15) as

$$
\left(J_{\Theta}^{m}\right)_{\alpha}=\left(\Theta\left(\gamma^{n} \partial_{n} X^{a^{\prime}} \gamma_{a^{\prime}}-\frac{1}{3} h^{n_{1} n_{2} n_{3}} \gamma_{n_{1} n_{2} n_{3}}\right) \gamma^{m}\right)_{\alpha}
$$

The full non-linear form of this current can be read off from the supersymmetry transformation given in [2] and [4, 3].

It is straightforward to verify that these currents do indeed lead to generators that satisfy the above worldvolume superalgebra.

\section{$5 \quad$ Automorphisms of Superalgebras}

Let us consider a supersymmetry algebra of the form

$$
\left\{Q_{\underline{\alpha}}, Q_{\underline{\beta}}\right\}=Z_{\underline{\alpha} \beta}, \quad\left[Q_{\underline{\gamma}}, Z_{\underline{\alpha} \beta}\right]=0, \quad\left[Z_{\underline{\alpha} \underline{\beta}}, Z_{\underline{\gamma} \underline{\delta}}\right]=0,
$$

where $Z_{\underline{\alpha} \beta}$ are Grassmann even generators labelled by spinor indices. It is obviously symmetric in these indices. Clearly, such an algebra obeys the generalized super Jacobi identities. Let us assume that the generators $Z_{\alpha \underline{\beta}}$ form the most general symmetric matrix. Expanding this matrix out in terms of the relevant Cliffor $\bar{d}$ algebra we find that $Z_{\underline{\alpha} \beta}$ contains a set of totally antisymmetric tensorial generators which constitute the central charges including the momentum generator. This is the case for both the eleven dimensional and the six dimensional superalgebras associated with the fivebrane equations of motion (11) to (3). Clearly in the latter case one must take an appropriate index range for the indices $\underline{\alpha}, \underline{\beta} \ldots$. The corresponding superalgebras in the IIA and IIB theories for which the $Z_{\underline{\alpha} \beta}$ form the most general matrix in ten dimensions are well known.

We now want to consider the automorphism group of such a superalgebra. Let $R$ be one of the generators of this group. Being Grassmann even its commutator with the supercharges must be of the form

$$
\left[Q_{\underline{\alpha}}, R\right]=M_{\underline{\alpha}} \underline{\beta} Q_{\underline{\beta}} .
$$

The super Jacobi identities then imply that

$$
\left[Z_{\underline{\alpha} \underline{\beta}}, R\right]=M_{\underline{\alpha}} \underline{\underline{\gamma}} Z_{\underline{\gamma \beta}}+M_{\underline{\beta}} \underline{\gamma} Z_{\underline{\alpha} \underline{\gamma}}
$$

It is straightforward to verify that all the remaining super Jacobi identities are satisfied provided the matrices $M$ form a representation of the Lie algebra generated by the generators $R$.

Clearly, the maximal automorphism group is $G L\left(c_{d}\right)$ where $c_{d}$ is the number of supercharges. The precise properties of the matrices under complex conjugation being given by implementing the Majorana or other properties of the spinorial supercharge on the commutator relation of the supercharge with the automorphisms. The generators may be labelled as $R_{\underline{\underline{\gamma}}} \underline{\text { and }}$ their commutator with the supercharges is given by $\left[Q_{\underline{\alpha}}, R_{\underline{\gamma}} \underline{\delta}\right]=\delta_{\underline{\alpha}} \underline{\delta} Q_{\underline{\gamma}}$. To gain a more familiar set of generators we may expand $R_{\underline{\underline{\gamma}}} \underline{\delta}$ out in terms of the elements of the enveloping Clifford algebra

$$
R_{\underline{\gamma}}^{\underline{\delta}}=\sum_{p} \sum_{\underline{a}_{1} \cdots \underline{a}_{p}}\left(\underline{\gamma}^{\underline{a}_{1} \ldots \underline{a}_{p}} C^{-1}\right)_{\underline{\gamma}}^{\underline{\delta}} R_{\underline{a}_{1} \cdots \underline{a}_{p}} .
$$


An interesting subalgebra is given by the set of symmetric matrices, these form Lie algebra of $S p\left(c_{d}\right)$. The generators of this subalgebra are $S_{\underline{\gamma} \underline{\delta}}=R_{\underline{\gamma} \underline{\delta}}+R_{\underline{\delta} \underline{\gamma}}$ where $R_{\underline{\gamma} \underline{\delta}}=R_{\underline{\gamma}} \underline{\underline{\beta}}\left(C^{-1}\right)_{\underline{\beta} \underline{\delta}}$. Clearly, in the decomposition of equation (44) this means keeping only those terms for which the symmetric matrices enter. In eleven dimensions these are the generators $R_{\underline{a}_{1} \ldots \underline{a}_{p}}$ of ranks one, two and five. The commutator of the generators $S_{\underline{\gamma} \underline{\delta}}$ with the supercharges is given by

$$
\left[Q_{\underline{\alpha}}, S_{\underline{\gamma} \delta}\right]=\left(C^{-1}\right)_{\underline{\alpha} \delta} Q_{\underline{\gamma}}+\left(C^{-1}\right)_{\underline{\alpha} \underline{\gamma}} Q_{\underline{\delta}} .
$$

One can also consider the case where $Z_{\alpha \underline{\beta}}$ is not the most general symmetric matrix. For example, when it is of the form $P \underline{a}\left(\Gamma_{\underline{a}}\right)_{\underline{\alpha} \underline{\beta}}$, implying that we are dealing with point particles only. Then the most general automorphism group is by definition the group $\operatorname{Spin}(1, d-1)$. These are the generators $R_{\underline{a}_{1} \underline{a}_{2}}$ in the decomposition of equation (44).

Including further central charges one finds a natural generalization of the spin group that takes into account the presence of branes. It would be interesting to compute the relevant spin group generalizations corresponding to removing various sets of central charges from the full possible set.

Clearly the generators of the generalized spin group will rotate the central charges into one another. As an example let us consider how a generator of $G L\left(c_{d}\right)$ rotates the central charges. To be concrete, let us consider the eleven dimensional supersymmetry algebra of equation (28) and consider the effect of the generators $R_{\underline{a}_{1} \underline{a}_{2} \underline{a}_{3}} \in G L(32)$, not belonging to $\operatorname{Sp}(32)$. One finds that

$$
\begin{aligned}
& {\left[P_{\underline{b}_{1}}, R^{\underline{a}_{1} \underline{a}_{2} \underline{a}_{3}}\right]=-12 \eta_{\underline{b}_{1}}^{\underline{a}_{1}} Z^{\underline{a}_{1} \underline{a}_{2}},} \\
& {\left[Z_{\underline{b}_{1} \underline{b}_{2}}, R^{\underline{a}_{1} \underline{a}_{2} \underline{a}_{3}}\right]=2 \eta \underline{\underline{b}}_{1} \underline{\underline{a}}_{2} \underline{\underline{a}}_{2} P^{\underline{a}_{3}}-5 ! Z_{\underline{b}_{1}} \underline{\underline{a}}_{1} \underline{\underline{a}}_{2} \underline{a}_{3},}
\end{aligned}
$$

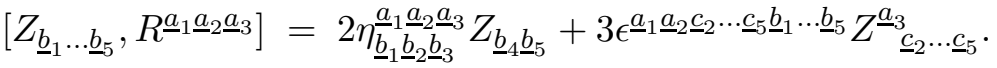

Here antisymmetry in $\underline{a}_{1} \underline{a}_{2} \underline{a}_{3}$ is to be understood and $\left.\eta_{\underline{b}_{1} \ldots \underline{a}_{n}} \ldots \underline{a}_{n} \eta_{\underline{b}_{1}} \ldots \underline{a}_{1} \ldots \underline{\underline{b}}_{n}\right]$.

Since the central charges are the topological charges for the corresponding branes [9] we must conclude that the automorphism group must rotate the different branes into each other.

\section{New Brane Symmetries}

In this section we will show that at least a part of the generalised spin group, or automorphism group, discussed in the previous section is realised as a symmetry of the fivebrane equations of motion. In particular we will find the associated new current and find the Poisson bracket relations of its generators with the central charges. For simplicity we will keep working with a flat background and also ignore terms of higher order in the Fermions.

Consider the following current

$$
R_{3}^{m \underline{a b c}}=\sqrt{-g}\left(* H^{m n p} \nabla_{n} X^{\underline{a}} \nabla_{p} X^{\underline{b}} X^{\underline{c}}+\frac{1}{2 e e e} \epsilon^{m n p q r s} \nabla_{n} X^{\underline{a}} \nabla_{p} X^{\underline{b}} \nabla_{q} X^{\underline{c}} B_{r s}\right)
$$

where anti-symmetry in $\underline{a b c}$ is assumed. To check that it is conserved recall the following facts

$$
\nabla_{m} * H^{m n p}=0, \quad H_{m n p}=3 \nabla_{[m} B_{n p]} .
$$

Note that (47) can be rewritten as

$$
R_{3}^{m \underline{a b c}}=\partial_{n}\left(\frac{1}{2} \sqrt{-g} \epsilon^{m n p q r s} \nabla_{p} X^{\underline{a}} \nabla_{q} X^{\underline{b}} X^{\underline{c}} B_{r s}\right),
$$


from which conservation is obvious.

Given this additional current we can use the same procedures as earlier and compute various Poisson brackets at lowest order. To make our notation less cumbersome we will use the same symbol to denote the associated conserved charge.

We now calculate the commutators of the $R_{3}^{a b c}$ with the central terms. Using the Poisson brackets of equations (24) we find that

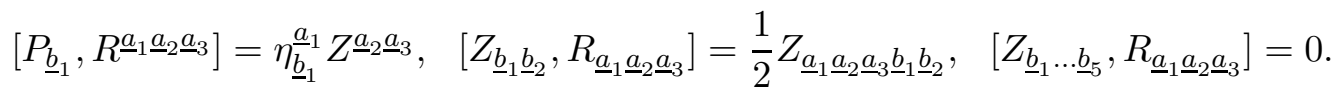

Up to normalisation this is a contraction of the algebra of equation (46) obtained by scaling the generators as follows

$$
P^{\underline{a}} \rightarrow P^{\underline{a}}, \quad R_{\underline{a}_{1} \underline{a}_{2} \underline{a}_{3}} \rightarrow \frac{1}{e} R_{\underline{a}_{1} \underline{a}_{2} \underline{a}_{3}} Z^{\underline{a}_{1} \underline{a}_{2}} \rightarrow \frac{1}{e} Z^{\underline{a}_{1} \underline{a}_{2}}, \quad Z^{\underline{a}_{1} \cdots \underline{a}_{5}} \rightarrow \frac{1}{e^{2}} Z^{\underline{a}_{1} \cdots \underline{a}_{5}} .
$$

and letting $e \rightarrow 0$. It is conceivable that the contraction is the effect of our approximation and that by carrying out the calculation for the full theory one may find the commutation relations of the full automorphism group.

\section{Conclusions}

We have shown, in the context of the covariant equation of motion, that the fivebrane possesses symmetries that lead to the worldvolume supersymmetry algebra and the eleven dimensional supersymmetry algebra. We have explicitly identified the corresponding currents by writing the equations of motion as total derivatives. In addition to the obvious currents for translations and supersymmetry we find that there exists a conserved current associated with the second rank tensor gauge field of the fivebrane. The generators of these currents lead to shift symmetries for all the fields of the fivebrane so allowing us to identify them as Goldstone fields for these symmetries.

Motivated by the observation that both eleven dimensional and worldvolume superalgebras associated with the fivebrane have central charges that constitute the most general symmetric matrix on the right hand-side of the anti-commutator of two supercharges we show that the automorphism group of such a generic superalgebra is $G L\left(c_{d}\right)$ where $c_{d}$ is the number of supercharges. This is the natural generalisation of the spin group which occurs in point particle theories to a theory, such as M theory, that possess branes as well as point particles. Such an automorphism group must rotate the different branes into one another and so is a generalisation of the previously known duality symmetries.

We show, at lowest order, that part of this automorphism group is realised as a new symmetry of the fivebrane equations of motion. At first sight, it may appear as a puzzle that this new brane rotating symmetry can be seen solely within the context of the fivebrane. However, the fivebrane through its equations of motion can see many of the effects in the eleven dimensional superspace including the presence of twobranes. Indeed, the twobranes which intersect fivebranes induce strings on the fivebrane whose charge is measured by the gauge field of the fivebrane. Examining the new current we find that the gauge field plays the central role in its construction consistent with its brane rotating property.

As such, it is natural to speculate that the $G L(32)$ automorphism group of the eleven dimensional supersymmetry algebra, or a subgroup of it, is a symmetry of M theory when properly formulated. Incorporating this symmetry would inevitably lead to a formulation in which the fivebrane and twobrane appeared on an equal footing. Since the symmetry rotates the twobrane and the fivebrane into each 
other one may wonder if it is a hidden symmetry of the eleven dimensional supergravity that rotates the corresponding brane solutions into one another. In the quantum theory the topological charges of the branes obey a generalisation of the Dirac quantization condition and so in the quantum theory one should only attempt to incorporate the subalgebra of the automorphism group that preserves this relation.

The new symmetries we have found are not just restricted to the branes of $\mathrm{M}$ theory, but by reducing the fivebrane to the $\mathrm{D} 4$ brane in ten dimensions and then using $\mathrm{T}$ duality we can extend it to be relevant to all the branes in ten dimensions.

Acknowledgment: We would like to thank Neil Lambert for helpful discussions. While this work was being written up we learned from Garry Gibbons that he, Jerome Gauntlett, Chris Hull and Paul Townsend were preparing a paper in which the automorphism group of superalgebras is considered within the context of BPS states and, in particular, those that preserve $3 / 4$ supersymmetry.

\section{References}

[1] O. Bärwald, N. D. Lambert, and P. C. West. A calibration bound for the M-theory fivebrane. Phys. Lett., B463:33-40, 1999. hep-th/9907170.

[2] O. Bärwald, N. D. Lambert, and P. C. West. The energy momentum tensor of the M-theory fivebrane. Phys. Lett., B459:125-133, 1999. hep-th/9904097.

[3] J. P. Gauntlett, N. D. Lambert, and P. C. West. Branes and calibrated geometries. Commun. Math. Phys., 202:571, 1999. hep-th/9803216.

[4] P. S. Howe, N. D. Lambert, and P. C. West. The Self-Dual String Soliton. Nucl. Phys., B515:203216, 1998. hep-th/9709014.

[5] P. S. Howe, N. D. Lambert, and P. C. West. The three-brane soliton of the M-five-brane. Phys. Lett., B419:79, 1998. hep-th/9710033.

[6] P. S. Howe, E. Sezgin, and P. C. West. Covariant field equations of the M theory five-brane. Phys. Lett., B399:49-59, 1997. hep-th/9702008.

[7] P. S. Howe, E. Sezgin, and P. C. West. The six dimensional self-dual tensor. Phys. Lett., B400:255259, 1997. hep-th/9702111.

[8] D. Sorokin and P. K. Townsend. M theory superalgebra from the M five-brane. Phys. Lett., B412:265, 1997. hep-th/9708003.

[9] J. A. de Azcarraga, J. P. Gauntlett, J. M. Izquierdo and P. K. Townsend. Topological extensions of the superalgebra for extended objects. Phys. Rev. Lett., B63:2443, 1989. 